\title{
Resistance Bridge Based on the Cryogenic Current Comparator in a Transport Dewar
}

\author{
E. Bartolomé, A. Camón, J. Sesé, C. Rillo, J. Flokstra, G. Rietveld and H. Rogalla
}

\begin{abstract}
An optimized resistance bridge based on the Cryogenic Current Comparator (CCC) with SQUID readout has been developed. The bridge can be used for routine secondary resistance calibrations as well as for the traceability of $100 \Omega$ to the quantum Hall resistance. The CCC-SQUID, shielding and wiring are mounted in a telescopic insert, fitted in a low-evaporation rate, transportable liquid He dewar, with an autonomy of around 1 month. A difference compensator method is used to balance the bridge. A new, more reliable and self-standing bridge electronics has been fabricated and tested. An uncertainty in the comparison of resistors at least one order of magnitude better than room temperature CCs is expected.
\end{abstract}

Index Terms-Cryogenic Current Comparator, Electrical quantum metrology, resistance bridge, SQUIDs.

\section{INTRODUCTION}

$\mathrm{T}$ HE CCC with SQUID readout is the most accurate system for the comparison of standard resistors, and traceability to the quantum Hall resistance (QHR). Given the complexity and high cost of these systems, practically all CCC based bridges are owned by National Metrology Institutes (NMIs) [1]-[4]. Officially, the calibration of primary resistors to the QHR has to take place at NMIs. However, the existence of a simple $\mathrm{CCC}$ for routine secondary resistance calibrations at industry and other institutes would be very desirable. These are the requirements that such a system should fulfill: i) the cryogenic system should be of small size, transportable, have a low evaporation rate and be easy to refill, ii) different ratios should be available for versatility, with no need to warm up the system, iii) the bridge electronics should be reliable, protected against environmental noise and able to work on batteries for a long time, iv) an accuracy at least one order of magnitude better than that of room temperature CCs $\left(<10^{-7}\right)$, and finally, v) the system should be easy to handle outside of the community of specialists. This paper describes the development and test of such a CCC bridge.

Manuscript received September 19, 2000. This wotk has been supported by the Spanish Ministry of Education(CICYT, MAT98-0668), the "technologiestichting STW", and the NMi, The Netherlands.

E. Bartolomé, J. Flokstra and H. Rogalla are with the Low Temperature Division, Dept. of Applied Physics, University of Twente; P.O. Box 217 , 7500AE Enschede, The Netherlands (telephone: 31-53-4343125; e-mail: e.bartolome@tn.utwente.nl).

A. Camón, C. Rillo and J. Sesé are with the ICMA, CSIC-University of Zaragoza, 50009 Zaragoza, Spain (e-mail: crillo@posta.unizar.es)

G. Rietveld is with the NMi Van Swinden Laboratorium, Dept. Electricity and Magnetism, Delft, The Netherlands (e-mail: grietveld@nmi.nl)

\section{THE CCC RESISTANCE BRIDGE}

A general picture of the $\mathrm{CCC}$ resistance bridge is shown in Fig. 1.

\section{A. Cryogenic insert}

The $\mathrm{CCC}$ is mounted at the end of a telescopic probe, immersed in a 120 litres, $7 \mathrm{~cm}$ neck transportable Dewar. A $\mathrm{Pb}$ cylindrical shield surrounds the CCC-SQUID to avoid the influence of external magnetic fields. The telescopic mechanism allows cooling down the system very slowly, with low evaporation of liquid helium $(\sim 0.5$ l) and no necessity to pre-cool with nitrogen. Any combination of the different $\mathrm{CCC}$ windings to obtain the desired ratios can be easily made in a connection box placed at the top of the insert. This cryogenic system allows around 1 month of continuous operation.

\section{B. CCC-SQUID system}

The core of the bridge is a type I CCC, containing the following windings: $1,1,2,4,16,16,32,64,128,465,800$

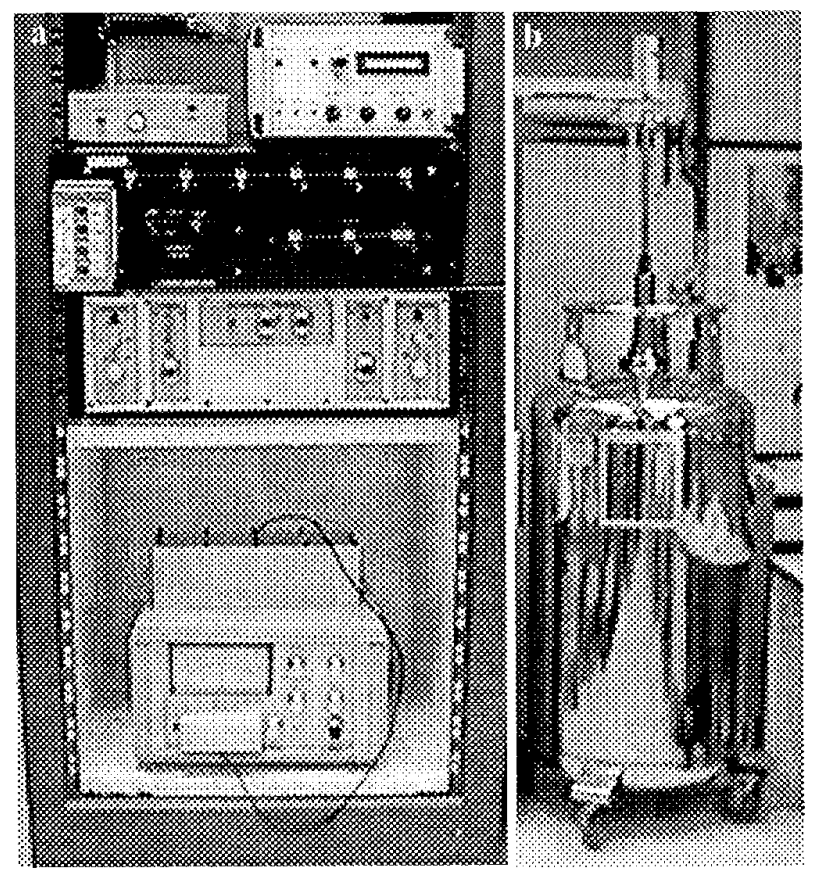

Fig. 1. Overview of the $\mathrm{CCC}$ based resistance bridge. a) Bridge electronics (from top to bottom): integrator, SQUID readout, "difference compensator"; current sources, two test $100 \Omega$ resistors and nano-voltmeter; b) transportable Dewar with tclescopic insert. 

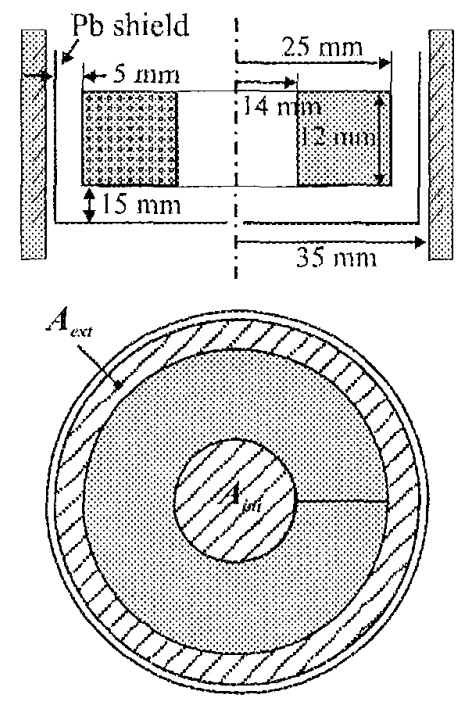

Fig. 2. Design of the CCC. Top; cross-section; Bottom: top view.

and 800 , that allow obtaining the ratios 1:1, 1:10, 1:100, $\mathrm{i}_{2}: 100 \Omega$ and $\mathrm{i}_{4}: 100 \Omega, \quad\left(\mathrm{i}_{2}\right.$ and $\mathrm{i}_{4}$ are respectively the resistances of the $2^{\text {nd }}$ and $4^{\text {th }}$ plateaus of the QHR). The windings are made in superconducting wire $(70-\mu \mathrm{m}$ monofilament $\mathrm{NbTi}$ with copper matrix), to avoid leak resistance. In order to reduce the input current noise $\left(I_{\mathrm{n}}\right)_{\mathrm{CCC}} \mathrm{N}_{\mathrm{CCC}}$, the CCC overlapped tube inductance $L_{\mathrm{CCC}}$ should be as large as possible. $L_{C C C}$ increases approximately linearly with the diameter of the $\mathrm{CCC}$ toroid, but is reduced to an effective value $L_{\mathrm{CCC}}$, eff when the distance between the CCC external wall approaches the lead magnetic shield, due to the image effect [5]. The neck of the Dewar is the maximum available for low-evaporation rate, transportable cryostats $(7 \mathrm{~cm})$. Since some lateral space has to be left for mounting the shield, the external radius of the $\mathrm{CCC}$ overlapped tube was limited to $D_{\mathrm{ext}} \sim 50 \mathrm{~mm}$. In order to have the maximal $L_{C C C}$, eff, the area enclosed by the $\mathrm{CCC}$ and the area comprised between the $\mathrm{CCC}$ external wall and shield should be equal (Fig. 2): $A_{\text {int }}=A_{\text {ext }}$.

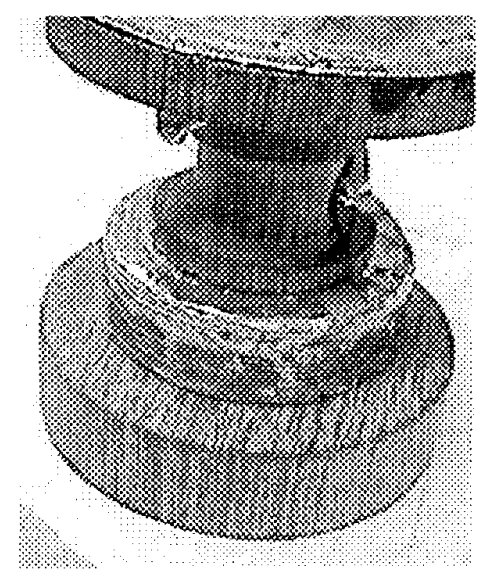

Fig. 3. CCC mounted on the celotex support; the sensing coil, wound around the overlapped tube, is visible
With this condition, the $\mathrm{CCC}$ internal diameter was set $d_{\mathrm{i}} \sim 28 \mathrm{~mm}$. The CCC height is $h \sim 12 \mathrm{~mm}$ to accommodate all the windings. The lateral and bottom distances between the $\mathrm{CCC}$ and the $\mathrm{Pb}$ shield are $5 \mathrm{~mm}$ and $15 \mathrm{~mm}$, respectively. The upper shield cover is sufficiently far from the $\mathrm{CCC}$, so that its shielding effect can be neglected. With these dimensions, the $L_{\mathrm{CCC}}$, eff simulated with the Quickfield finite element program as described in Ref. [6], was $\sim 17 \mathrm{nH}$. The CCC tube was made of $0.125 \mathrm{~mm}$-thick, high purity $(99.95 \%)$ lead to avoid the presence of magnetic impurities. The toroid is overlapped 3 and half times over itself, to minimize flux leakage. The isolator used between the different overlapping turns was kapton.

The SQUID is a commercial Oxford rf SQUID ("model $\left.\Phi_{0} "\right)$, with an input coil inductance of $L_{i}=1.3 \mu \mathrm{H}$, mutual inductance of $M_{\mathrm{sq}}=15 \mathrm{nH}$, intrinsic energy resolution of 9 $\mathrm{x} 10^{-29} \mathrm{~J} / \mathrm{Hz}$ (in the white region) and a corner frequency $<$ $0.1 \mathrm{~Hz}$. Due to the mismatch between $L_{i}$ and $L_{C C C \text {,eff, a }}$ sensing coil is required to couple the maximum flux of the CCC into the SQUID. A $N_{s}=8$ turn sensing coil is tightly wound around the CCC external wall. This configuration allows a better coupling than if the sensing coil is placed at the inner side of the CCC, thus leading to a better sensitivity [5]. The SQUID module is placed inside the toroid, and the whole ensemble mounted in a celotex support that provides mechanical rigidity (Fig. 3 ).

\section{CCC bridge electronics}

A schematic of the resistance bridge developed is shown in Fig. 4. A primary current $I_{\mathrm{p}}$ created by the primary current source $\mathrm{S}_{\mathrm{p}}$ feeds the reference resistance $R_{\mathrm{p}}$ and the CCC winding $\mathrm{N}_{\mathrm{p}}$. A secondary current $I_{\mathrm{s}}$, generated by a secondary current source $S_{s}$ (slave of $S_{p}$ ), feeds the resistance $R_{s}$ to be compared, and the $\mathrm{CCC}$ winding $\mathrm{N}_{\mathrm{s}}$. A voltage ramp generator feeds the two voltage controlled current sources, so that the polarity, magnitude and slope of the two currents can be inverted simultaneously. The two currents traverse $R C$ filters to reduce the noise coupling to the SQUID. The values of $R$ and $C$ can be adjusted to maintain the filter frequency constant when a different resistance $R_{\mathrm{s}}$ is placed. A first, coarse adjustment (parts in $10^{5}$ ) of the bridge is realized by a manual tracking system, leaving the fine adjustment (parts in $10^{10}$ ) to the SQUID feedback loop. In this way the SQUID does not have to cope with large signal variations, and remains locked. The isolation stage provides an isolation better than $10^{13} \Omega$ between the two branches of the bridge. An EM, N1l nano-voltmeter measures the difference in the voltage across the two resistors. It can be connected to an $8^{1 / 2}$ digits multimeter (HP3458A) and be readout as a function of time with a computer. The unbalance between the two currents produces a Meissner current $l_{\mathrm{e}}$ in the overlapped tube of the CCC described in section $B$. The flux $\Phi_{e}$ created by this current is coupled into the rf SQUID. The Oxford SQUID electronics feeds back the secondary source via a fiber optic stage that isolates perfectly 


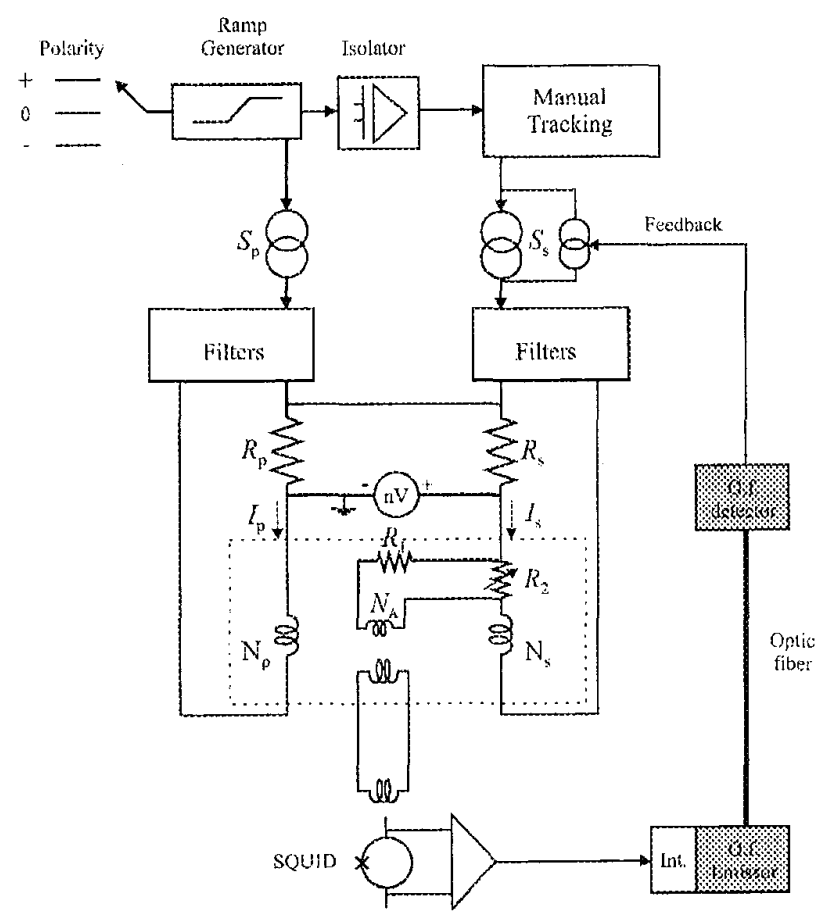

Figure 4. Schematic of the CCC resistance bridge electronics.

the two electronics, avoiding the large amount of noise coupling into the SQUID when a coaxial cable is used. An integrating stage in the feedback loop was designed to compensate for the filter pole and fix the system cut-off frequency at $\sim 110 \mathrm{~Hz}$, while keeping a high gain $\left(>10^{5}\right)$. With these precautions, the feedback loop will be stable independent of the ratio of windings chosen. The whole system is fed by $\mathrm{NiCd}$ batteries to avoid interference from power grid. Each part of the system is closed in separately guarded boxes to minimize electronic cross-talk. The bridge is grounded at the $\mathrm{V}^{-}$input of the nano-voltmeter.

\section{Characteristics of THE CCC-SQUID}

The main characteristics of the newly built CCC-SQUID system have been measured. The winding ratio error due to flux leakage in the overlapped tube was determined passing a $4 \mathrm{~mA}$ current through the series-opposition connection of two 800 turn windings.

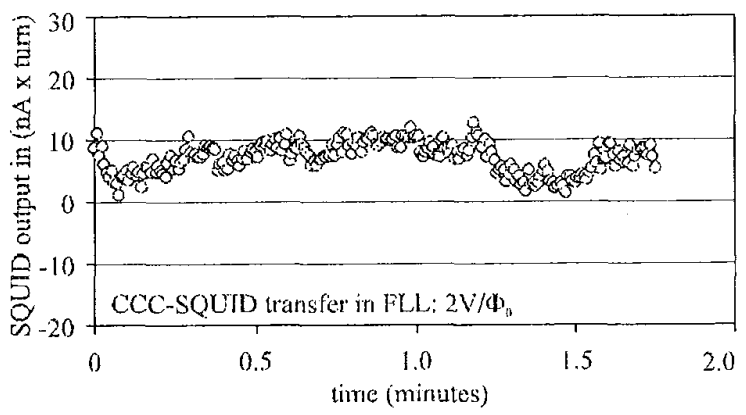

Fig. 5. Zero stability of the CCC-SQUID.
It was smaller than parts in $10^{9}$ and limited by the SQUID noise. The sensitivity of the system, measured passing a $20 \mu \mathrm{A}$ current through a 16-turn winding, was $4 \mu \mathrm{A} x$ turn $/ \Phi_{0}$. The zero stability of CCC-SQUID was measured recording the SQUID output as a function of time with no currents applied to the windings (Fig. 5). The linear drift is small $(-0.38 \mathrm{nA} \times$ turns/min), and the current resolution found was $\sigma=2.4 \mathrm{nA} \times$ turn.

\section{DIFFERENCE COMPENSATOR METHOD}

The feedback of the CCC bridge guarantees that $N_{\mathrm{p}} I_{\mathrm{p}}=N_{\mathrm{s}} I_{\mathrm{s}}$. A nano-voltmeter is used to measure the difference in voltage drop across the two resistors, $\Delta V$. When the two resistances are exactly in the same ratio as the two windings: $R_{\mathrm{s}}=\left(N_{\mathrm{s}} / N_{\mathrm{p}}\right) R_{\mathrm{p}}$, then $\Delta V=0$. A deviation $\Delta R_{\mathrm{s}}$ of the secondary resistance from its nominal value, will produce a proportional voltage signal $\Delta V$, thus: $\Delta R_{\mathrm{s}}=\Delta V / I_{\mathrm{s}}$. Since the secondary current $I_{\mathrm{s}}$ is not known with high accuracy, a calibration method is needed. An earlier version of the bridge [7] used a calibration pulse method to obtain the value of $\Delta R_{\mathrm{s}}$. A resistor, $R_{\mathrm{cp}}$ shunts $R_{\mathrm{s}}$ for a short time, so a known resistance variation of e.g. $1 \mathrm{ppm}$ is introduced. A voltage pulse $V_{\text {cp }}$ is produced, which can be used to scale the value of $\Delta V$ and obtain $\Delta R_{\mathrm{s}}$. This calibration method presents several problems. First, in order to scale the $\Delta V$ with $V_{\mathrm{cp}}$, both voltages must be read in the same scale, and since $V_{\text {cp }} \gg \Delta V, \Delta V$ is obtained with poor resolution. Besides, the sudden variation in the bridge introduced by the pulse easily unlocks the SQUID.

An alternative, "difference compensator" method [8] has been implemented to solve these problems. It consists of deviating a known part of the secondary current $I_{\mathrm{s}}$ through an auxiliary $\mathrm{CCC}$ winding $N_{\mathrm{A}}$, owing to a current divider composed of $R_{1}$ and $R_{2}$ (Fig. 4), such that: $I_{\mathrm{\Lambda}}=R_{2} I_{\mathrm{s}} /\left(R_{1}+R_{2}\right)$. $R_{1}$ and/or $R_{2}$ can be varied so as to balance the bridge. The secondary resistor will be:

$$
R_{s}=R_{p} \frac{N_{s}}{N_{p}}(1-\varepsilon) \text {, where } \varepsilon=\frac{N_{A}}{N_{s}} \frac{R_{2}}{R_{1}+R_{2}}
$$

Since the nano-voltmeter works as null detector, no error due to the non-linearity of the instrument is introduced. In addition, no sudden change is introduced in the bridge, so the feedback loop remains closed. The accuracy with which the resistors $R_{1}$ and $R_{2}$ must be known depends on the value of $\varepsilon$. For instance, if $\varepsilon=1 \mathrm{ppm}$, in order the error committed by the difference compensator to be less than 1 part in $10^{8}$, we should know $R_{1}$ and $R_{2}$ with $1 \%$ accuracy. It is not necessary to achieve a perfect zero in the nano-voltmeter to deduce the exact value of $R_{1}$ and $R_{2}$ that balance the bridge. It is enough to approach as much as possible to zero and calculate $R_{2} /\left(R_{1}+R_{2}\right)$ by interpolation. As a consequence, it is not necessary to control $\varepsilon$ to parts in $10^{8}$.

The new CCC bridge utilises the difference compensator method that we have tested on the existing prototype bridge [9]. We compared two resistors with nominal value 100 $\Omega$, using two $\mathrm{N}_{\mathrm{s}}=\mathrm{N}_{\mathrm{p}}=64$-turn windings, and an auxiliary 


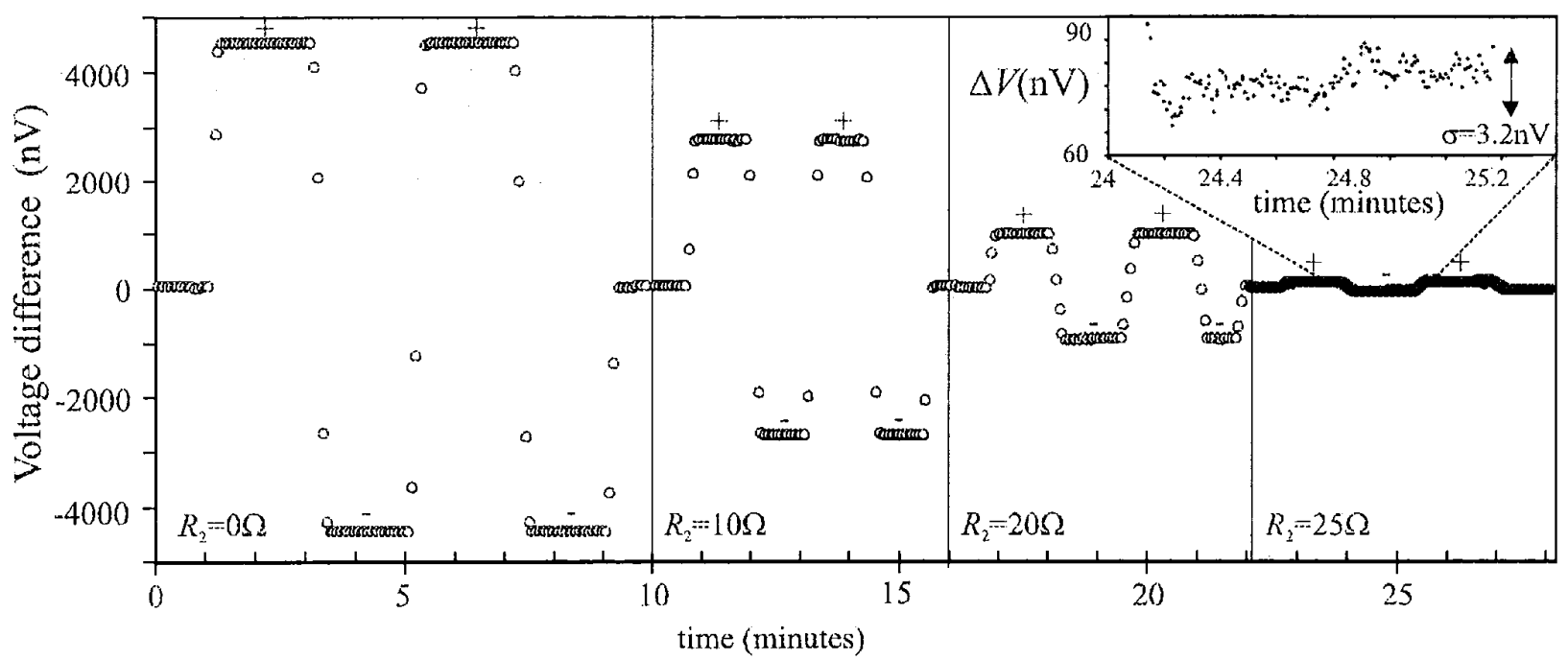

Fig. 6. Voltage $\Delta V$ in the nano-voltmeter as a function of time for three different values of the adjustable resistor $R_{2}$. The polarity change in $I_{\mathrm{p}}(0,+,-,+, 0)$ is indicated. The inset shows the stability within a plateau.

$\mathrm{N}_{\mathrm{A}}=16$ turn-winding, $R_{1}$ was a precision resistor with fixed value $R_{1}=4.9999026 \mathrm{M} \Omega$, and the variable $R_{2}$ had an accuracy of a few parts in $10^{4}$. We made four automated measurements, with the following values of $R_{2}: 0,10,20$ and $25 \Omega$. In each $\sim 6$ minutes run, the primary current $I_{\mathrm{p}}$ was switched from 0 to alternatively,,,$+-+-4.18 \mathrm{~mA}$. Results are shown in Fig. 6. The voltage standard deviation is $\sigma=3.2$ $\mathrm{nV}$ (inset Fig. 6). The value of $R_{2}$ compensating exactly the bridge, obtained by interpolation (Fig. 7), is $R_{2}=25.44 \pm$ $0.03 \Omega$. Therefore, the difference of the secondary resistor with respect to its nominal value is found to be: $\Delta R_{\mathrm{s}} / R_{\mathrm{s}}=2.544 \mathrm{ppm}$, with an uncertainty of $8 \mathrm{ppb}$.

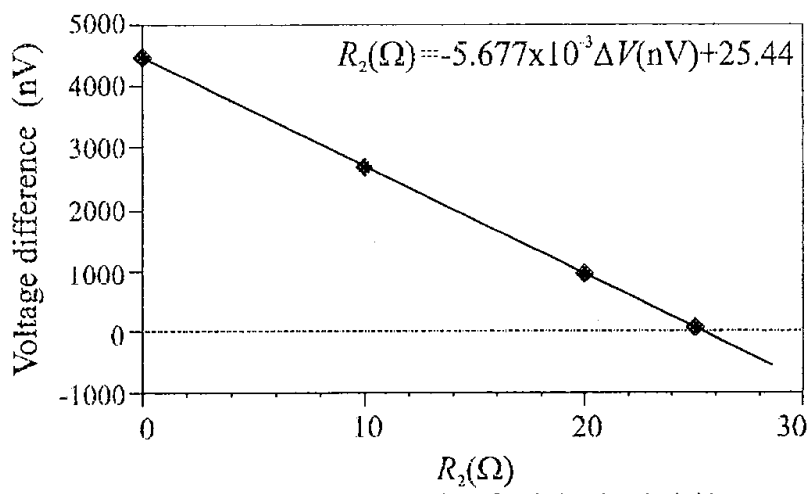

Fig. 7 Interpolation to obtain the exact value of $R_{2}$ balancing the bridge.

\section{CONCLUSIONS}

An optimized resistance bridge based on the CCC-SQUID, with a user-friendly, transportable cryogenic system has been developed.
The relative simplicity, low cost and better uncertainty level makes the instrument competitive respect to today's more extended room temperature current comparators. Using the difference compensator method, type A uncertainty levels of $8 \mathrm{ppb}$ have been achieved. The present system will be transferred to the TPYCEA Spanish secondary metrology institute.

\section{REFERENCES}

[1] J. M. Williams, P. Kleinschmidt, "A cryogenic current comparator for resistance measurements at currents up to $100 \mathrm{~A}^{\prime \prime}$, IEEE Trans. Instrum. Meas., vol. 48, 2, pp.375-378, 1999

[2] H. Seppä, A. Satrapinski, T. Varpula, J.M. Saari, "AC resistance Bridge based on the Cryogenic Current comparator", IEEE Trans. Instrum. Meas, vol. 46, 2, pp. 463-466, 1997

[3] E. Pesel, B. Schumacher, P. Warnecke, "Resistance scaling up to $1 \Omega$ at PTB with a cryogenic current comparator", IEEE Trans. Instrum. Meas., vol. 44, 2, pp.273-275, 1995 .

[4] F. Delahaye, "An AC Bridge for Low frequency measurement of the Quantized Hall Resistance", IEEE Trans. Instrum. Meas., vol. 40, 6, pp. 883-888, 1991.

[5] J. Sesé, A. Camón, C. Rillo and G. Rietveld, "Ultimate current resolution of a cryogenic current comparator", IEEE Trans. Instrum. Meas., vol. 48, 6, pp. 1306-1313, 1999.

[6] J. Sesé, F. Lera, A. Camón and C. Rillo, "Calculation of effective inductances of superconducting devices. Applicaton to the cryogenic current comparator", IEEE Trans. Appl. Supercond., vol. 9, 1, pp. 58-62, 1999

[7] A. Camón, PhD Thesis, University of Zaragoza, 1996.

[8] F. Delahaye and D. Reymann, "Progress in the Resistance ratio determination using a Cryogenic Current Comparator at LCIE", IEEE Trans. Instrum. Meas., vol. 34, 2, pp. 316-319, 1985

[9] J. Sesé, PhD Thesis, University of Zaragoza, 1999. 